\title{
Gender Differences in Body Composition in Pre-Frail Older Adults With Diabetes Mellitus
}

\author{
Reshma Aziz Merchant ${ }^{1,2 *}$, John Tshon Yit Soong ${ }^{2,3}$ and John E. Morley ${ }^{4}$ \\ ${ }^{1}$ Geriatric Medicine, Department of Medicine, National University Hospital, Singapore, Singapore, ${ }^{2}$ Department of Medicine, \\ Yong Loo Lin School of Medicine, National University Singapore, Singapore, Singapore, ${ }^{3}$ Advanced Internal Medicine, \\ Department of Medicine, National University Hospital, Singapore, Singapore, ${ }^{4}$ Division of Geriatric Medicine, Saint Louis \\ University School of Medicine, St Louis, MO, United States
}

\section{OPEN ACCESS}

Edited by:

Qing Feng Cheng,

First Affiliated Hospital of Chongqing

Medical University, China

Reviewed by:

Lunzhi Dai,

Sichuan University, China

Jirong Yue,

Sichuan University, China

*Correspondence: Reshma Aziz Merchant reshmaa@nuhs.edu.sg

Specialty section:

This article was submitted to Endocrinology of Aging, a section of the journal Frontiers in Endocrinology

Received: 15 October 2021 Accepted: 12 January 2022 Published: 15 February 2022

Citation:

Merchant RA, Soong JTY and Morley JE (2022) Gender Differences in Body Composition in Pre-Frail Older Adults With Diabetes Mellitus.

Front. Endocrinol. 13:795594. doi: 10.3389/fendo.2022.795594
Background \& Aims: Ageing is a risk factor for diabetes mellitus (DM) and frailty. It is associated with body composition changes including increase in fat mass (FM), central fat distribution, decrease in fat free mass (FFM) and skeletal muscle which are risk factors for DM. This study aims to evaluate gender differences in body composition in pre-frail diabetics and association with physical performance, cognitive function and perceived health. In addition, we aim to explore the association of obesity, sarcopenia, sarcopenic obesity, and body composition in pre-frail older adults to DM status.

Methods: Cross-sectional study of 192 pre-frail community dwelling older adults ( $\geq 65$ years). Data was collected on demographics, physical function, cognition, frailty, sarcopenia, perceived health and body composition using the InBody S10. Univariate and multivariate logistic regression were undertaken to explore the association of sarcopenic obesity, obesity, sarcopenia and body composition measures to DM status.

Results: There were insignificant within-gender differences for physical function, cognition and body composition, except for a higher prevalence of obesity defined by body mass index (BMI) and body fat percentage (BF\%), increased fat mass index(FMI) and fat free mass index(FFMI) in females with DM. There were significant between-gender differences for those with DM where females overall had lower education levels, lower perceived health, higher prevalence of depression and low mental vitality, lower overall physical function (low short physical performance battery scores, low gait speed and hand grip strength), lower cognitive scores, lower muscle mass and muscle quality with higher FMl, FM/FFM and visceral fat area(VFA). BMI, VFA $>100 \mathrm{~cm}^{2}$, FMI and FFMI were found to be independently associated with DM status after multivariable adjustment.

Conclusion: Within pre-frail DM vs non-DM, there were insignificant differences in body composition, physical function, cognition and perceived health within gender except for 
FMI, BF\% and FFMI in females. There were significant differences between gender in prefrail DM in muscle mass, quality, functional, cognitive and mental status. Further longitudinal studies are required to understand the pathogenesis, trajectory of DM and protective role of oral hypoglycemics in pre-frail older adults.

Keywords: pre-frailty, FRAIL scale, diabetes mellitus, body composition, physical function, cognitive function

\section{INTRODUCTION}

Population ageing is a global phenomenon and the number of older people $\geq 65$ years is projected to double to 1.5 billion in 2050 (1). Ageing is a risk factor for chronic diseases, and associated with increased prevalence of frailty, cognitive impairment, and obesity. Ageing is also associated with body composition changes including increase in fat mass (FM), ectopic fat distribution and visceral fat area (VFA), with decrease in fat free mass (FFM) and skeletal muscle which are all precursors of metabolic diseases including diabetes (2-5). Diabetes and its related complications including frailty, cognitive impairment and disability imposes a major threat to health and social care system worldwide. The International Diabetes Federation in 2019 estimated that $19.3 \% \geq 65$ years old worldwide had diabetes (6). A local study published in 2017 similarly showed the prevalence of diabetes was $23.8 \%$ amongst $\geq 65$ years old (7).

Ageing is associated with impaired glucose tolerance, increased prevalence of postprandial hyperglycemia and type 2 diabetes mellitus (DM). Physical inactivity, beta-cell dysfunction, decrease in skeletal muscle mass and increase in FM including visceral fat which has pro-inflammatory activity are thought to be the main predisposing factors for $\operatorname{DM}(8,9)$. BMI is often used as a predictor for DM, but it is becoming increasingly evident that BMI is not reliable especially in older adults and needs to be interpreted with caution as loss of physiological height with ageing may lead to over-interpretation $(3,10,11)$. Jo et al. showed that $64 \%$ of population $\geq 40$ years old with normal $\mathrm{BMI}$ had a high body fat percentage (BF\%), and prevalence of abnormal blood glucose was significantly higher in the normal $\mathrm{BMI}$ with high $\mathrm{BF} \%$ compared with overweight and low $\mathrm{BF} \%$ (12). BMI is a composite of FM and FFM and high BMI in older adults has been associated with better physical and cognitive function $(13,14)$. Recently body composition indicators such as waist-to-hip ratio, $\mathrm{BF} \%$, body fat mass and VFA have been found to be better predictors of type $2 \mathrm{DM}(15,16)$.

In recent years, frailty, pre-frailty, and sarcopenia have been found to be associated with increased adverse outcomes in older adults irrespective of underlying comorbidities. Frailty is a multidimensional concept and dynamic. It is defined as a state of decreased physiological reserve which increases vulnerability to adverse outcomes when exposed to stressors (17). Sarcopenia is a common cause of physical frailty and defined as age-related loss in muscle mass and strength with impact on physical performance (18). The prevalence of frailty amongst older adults with diabetes is almost double that of normal population, both share similar pathophysiology and frailty is a major factor associated with death and disability in diabetics and metabolic syndrome (19-22). The prevalence of frailty and prefrailty worldwide is between $50-60 \%$ and both are independent predictors of type 2 diabetes mellitus (7, 17, 23-25). While it is known that diabetics have lower muscle mass and high $\mathrm{BF} \%$, there is no data on body composition in pre-frail older adults with and without diabetes mellitus (26).

This study aims to evaluate gender differences in body composition in pre-frail diabetics and association with physical performance, cognitive function and perceived health. In addition, we aim to explore the association of obesity, sarcopenia, sarcopenic obesity, and body composition in prefrail older adults to DM status.

\section{MATERIALS AND METHODS}

This was a cross-sectional analysis of 192 pre-frail community dwelling older adults recruited for multidomain intervention from primary and community care in Singapore.

\section{Demographics and Covariates}

Trained research staff administered questionnaires covering demographics, chronic diseases, medications, falls, function, cognition, frailty, sarcopenia, depression, and perceived health. Multimorbidity was defined as $\geq 2$ self-reported chronic conditions. Mini nutritional Assessment tool was used to assess malnutrition risk (27). Activities of daily living (ADL) was assessed using Katz activity of daily living and instrumental activities of daily living (IADL) using Lawton's IADL scale (28, 29). Frailty was assessed using the FRAIL scale (Fatigue, Resistance, Aerobic, Illness, and Loss of Weight), where prefrail was defined based on scores of 1-2 (30). SARC-F was used to screen for sarcopenia (lifting and carrying 10 pounds, walking across a room, transferring from bed/chair, climbing a flight of 10 stairs, and frequency of falls in the past 1 year) with a range of 0-10 (31). Cognitive status was assessed using the Montreal Cognitive Assessment (MoCA) (32). The Rapid Assessment of Physical Activity (RAPA) tool was used to assess physical activity (33). Physically active was based on WHO recommendations of $\geq 150$ minutes of moderate intensity, or $\geq 75$ minutes of vigorous intensity aerobic physical activity per week (34). The 15-item Geriatric Depression Scale (GDS) was used to screen for depression with $\geq 5$ was used to define depression (35). Three questions from the GDS were used to define mental vitality: 1) Are you basically satisfied with your life? 2) Do you feel that your life is empty? 3) Do you feel full of energy? A score of zero defined high mental vitality while a score between one to three 
signified low mental vitality (36). EuroQol vertical visual analogue scale (EQ-VAS) was used to evaluate perceived health (37). DM status was self-reported by means of questionnaire.

\section{Physical Function, Waist Circumference and Calf Circumference}

Maximum handgrip strength (HGS), gait speed (GS) and Short Physical Performance Battery test (SPPB) was measured. Calf circumference (CC) was measured in seated position with feet on the floor and leg positioned at $90^{\circ}$ using non-elastic tape and taken at maximal circumference. HGS of the dominant hand was measured using Jamar hand dynamometer in the seated position with elbow flexed at $90^{\circ}$. Waist circumference (WC) was measured midpoint between the last rib and iliac crest. The SPPB included 3 components on balance, $4 \mathrm{~m}$ gait speed and chair stand with a maximum score of 12 points (4 points per-component).

\section{Body Composition and Body Mass Index}

BMI was calculated by dividing body weight $(\mathrm{kg})$ by height squared $\left(\mathrm{m}^{2}\right)$. Cut offs of $23 \mathrm{~kg} / \mathrm{m}^{2}$ and $27.5 \mathrm{~kg} / \mathrm{m}^{2}$ were used to define overweight and obesity respectively based on WHO recommendations for Asians (38). Underweight older adults defined as having a BMI of $<18.5 \mathrm{~kg} / \mathrm{m}^{2}$ were excluded. InBody S10, a multi-frequency bioelectrical impedance analyzer was used to estimate body composition including FM, FFM, body cell mass, appendicular skeletal muscle (ASM) and whole-body phase angle. Fat Mass Index (FMI) and Fat Free Mass Index (FFMI) were calculated as FM divided by height squared and FFM divided by height squared, respectively. Obesity was defined by $\mathrm{BF} \%>25 \%$ in males and $>35 \%$ in females (39). Normal muscle mass was defined based on to the 2019 AWGS criteria with ASM/height ${ }^{2}$ cut-offs of $\geq 7.0 \mathrm{~kg} / \mathrm{m}^{2}$ for males and $\geq 5.7 \mathrm{~kg} / \mathrm{m}^{2}$ for females (40). Sarcopenia diagnosis was based on the 2019 AWGS criteria of gender specific cut offs for ASM/height ${ }^{2}$ and either low HGS $(<28 \mathrm{~kg}$ for male and $<18 \mathrm{~kg}$ for female) or slow GS of $<1 \mathrm{~m} / \mathrm{s}$. Sarcopenic obesity was defined as having an ASM to BMI ratio of $<0.789$ and $\mathrm{BF} \%>25 \%$ in males or an ASM to BMI ratio of $<0.512 \& \mathrm{BF} \%>55$ in females (39). Muscle quality was defined based on HGS/ASMBMI (41).

\section{Statistical Analysis}

Within and between gender differences of the included participants for demographics, co-morbidity, anthropometrics, clinical assessments and bioimpedance analysis by DM status are represented in a frequency table. Categorical variables are presented as frequencies with percentages, while continuous variables are presented as mean with standard deviation. Significance testing by Chi-Square test for categorical and oneway ANOVA for continuous variables are presented.

Univariate and multivariate logistic regression was undertaken to explore the association of sarcopenic obesity, obesity, sarcopenia definitions, as well as body composition with DM status. Adjustment for weight loss, physical activity, gender, ethnicity, and age was undertaken for the multivariate regression. Odds ratios with confidence intervals, sensitivity, and specificity at Youden point and Area under the Receiver Operator Characteristic (ROC) Curve are presented. Statistical analysis was conducted using $\mathrm{R}$ Package version 4.1.0.

\section{Ethics Approval and Informed Consent}

The study conformed to the principles of the Declaration of Helsinki and was approved by The National Healthcare Group Domain Specific Review Board (Reference: 2017/00035 and 2018/01183). Informed consent was obtained from all participants.

\section{RESULTS}

Of the 192 pre-frail participants included in this study, 82(42.7\%) were male and $110(57.3 \%)$ female, with a mean age of 73 years \pm 5.7 years. The predominant ethnic group was Chinese $(82.8 \%)$ and average number of years in formal education was $7.8 \pm$ 4.3 years.

Within gender differences of participant characteristics are displayed in Table 1. In pre-frail older participants, BMI and WC was significantly higher in females with DM compared to non-diabetics (mean BMI of $27.3 \pm 4.3$ versus $25.2 \pm 3.8$; $\mathrm{p}=0.008$ and mean WC of $96.2 \pm 10.7 \mathrm{~cm}$ versus $89.8 \pm 12.2 \mathrm{~cm} ; \mathrm{p}=0.038$ respectively). This difference was not found between male diabetics and non-diabetics. Both male and female participants with DM had significantly increased multimorbidity compared to those with non-diabetics, with female participants with DM having significantly increased levels of hypertension and hyperlipidaemia compared to non-diabetics. Bioimpedance analysis revealed significant increases in FMI (mean $10.8 \pm 3.3$ versus $9.3 \pm 3.3$ ) and FFMI (mean $16.5 \pm 1.7$ versus $15.8 \pm 1.7$ ) in females with DM compared to non-diabetics. The prevalence of obesity defined by BF\% was significantly higher in females with DM compared with non-diabetics, $71.2 \%$ and $50.0 \%$ respectively. This difference was not found between male diabetics and non-diabetics.

Between gender differences of participant characteristics are displayed in Table 2. There was significantly lower education and perceived health in females with DM compared to males $(6.4 \pm 3.9$ versus $9.4 \pm 3.9$ years and EQ-VAS of $66.7 \pm 14.6$ versus $73.1 \pm 13.6$ respectively). There were significantly lower Rapid Assessment of Physical Activity scores but no difference in moderate physical activity levels in females compared with males with and without DM. The prevalence of low mental vitality was significantly higher in females compared with males regardless of underlying DM status (non-DM: $71.4 \%$ versus 44.4\%, DM: $66.0 \%$ vs $39.1 \%$ ). Females with DM had more than twice the prevalence of depression compared with males with DM, 38.0\% versus $17.4 \%$. Females had significantly lower MNA scores $(21.4 \pm 2.1$ versus $22.2 \pm 2.1)$. Overall, females compared with males with DM had slower chair times (mean $15 \mathrm{~s} \pm 5.7$ versus $12 \mathrm{~s} \pm 4.3)$, slower GS $(0.8 \pm 0.2 \mathrm{~m} / \mathrm{s}$ versus $1.0 \pm 0.3 \mathrm{~m} / \mathrm{s})$, lower $\mathrm{SPPB}$ scores $(8.9 \pm 2.3$ versus $10.4 \pm 2.5)$ and MoCA scores $(23.9 \pm 4.8$ versus $25.9 \pm 2.8)$. This difference was not found in 
TABLE 1 | Characteristics table displaying within gender differences by Diabetes Mellitus (DM) Status.

\begin{tabular}{|c|c|c|c|c|c|c|c|}
\hline & \multirow[t]{2}{*}{ All $(n=192)$} & \multicolumn{3}{|c|}{$\begin{array}{c}\text { Male } \\
82(42.7 \%)\end{array}$} & \multicolumn{3}{|c|}{$\begin{array}{c}\text { Female } \\
110(57.3 \%)\end{array}$} \\
\hline & & $\begin{array}{c}\text { No DM } \\
36(43.9 \%)\end{array}$ & $\begin{array}{c}\text { DM } \\
46(56.1 \%)\end{array}$ & $P$ value & $\begin{array}{c}\text { No DM } \\
58(52.7 \%)\end{array}$ & $\begin{array}{c}\text { DM } \\
52(47.3 \%)\end{array}$ & $P$ value \\
\hline \multicolumn{8}{|l|}{ Demographics } \\
\hline Age & $73.04 \pm 5.73$ & $74.39 \pm 6.74$ & $72.59 \pm 5.41$ & 0.183 & $72.52 \pm 5.50$ & $73.08 \pm 5.52$ & 0.596 \\
\hline Ethnicity & & & & 0.137 & & & 0.636 \\
\hline Chinese & $159(82.8)$ & 32 (88.9) & $33(71.7)$ & & $50(86.2)$ & $44(84.6)$ & \\
\hline Malay & $13(6.8)$ & $2(5.6)$ & $4(8.7)$ & & $4(6.9)$ & $3(5.8)$ & \\
\hline Indian & $19(9.9)$ & $2(5.6)$ & 9 (19.6) & & $3(5.2)$ & $5(9.6)$ & \\
\hline Others & $1(0.5)$ & & & & $1(1.7)$ & $0(0.0)$ & \\
\hline $\mathrm{BMI}\left(\mathrm{kg} / \mathrm{m}^{2}\right)$ & $25.89 \pm 4.17$ & $24.69 \pm 3.52$ & $26.00 \pm 4.57$ & 0.161 & $25.24 \pm 3.76$ & $27.33 \pm 4.33$ & 0.008 \\
\hline BMl status & & & & 0.895 & & & 0.019 \\
\hline Normal & $50(26.0)$ & $13(36.1)$ & $11(23.9)$ & & $16(27.6)$ & $10(19.2)$ & \\
\hline Overweight & $61(31.8)$ & $8(22.2)$ & $13(28.3)$ & & $14(24.1)$ & $26(50.0)$ & \\
\hline Obese & $81(42.2)$ & $15(41.7)$ & $22(47.8)$ & & $28(48.3)$ & $16(30.8)$ & \\
\hline Depression & $59(31.4)$ & $9(25.0)$ & $8(17.4)$ & 0.569 & $23(41.1)$ & $19(38.0)$ & 0.901 \\
\hline Hyperlipidaemia & $157(81.8)$ & $29(80.6)$ & $42(91.3)$ & 0.275 & $38(65.5)$ & 48 (92.3) & 0.002 \\
\hline Hypertension & $139(72.8)$ & $29(80.6)$ & $37(80.4)$ & 1 & $33(56.9)$ & $40(78.4)$ & 0.029 \\
\hline Multimorbidity & 161 (83.9) & $29(80.6)$ & $45(97.8)$ & 0.025 & $36(62.1)$ & $51(98.1)$ & $<0.001$ \\
\hline Education (years) & $7.84 \pm 4.27$ & $8.26 \pm 4.28$ & $9.39 \pm 3.88$ & 0.216 & $7.58 \pm 4.48$ & $6.40 \pm 3.93$ & 0.153 \\
\hline Calf circumference (cm) & $35.85 \pm 4.51$ & $36.17 \pm 3.55$ & $36.44 \pm 3.12$ & 0.728 & $34.93 \pm 3.69$ & $36.17 \pm 6.58$ & 0.23 \\
\hline Waist circumference (cm) & $93.05 \pm 10.93$ & $92.84 \pm 9.32$ & $94.93 \pm 9.75$ & 0.449 & $89.83 \pm 12.16$ & $96.18 \pm 10.74$ & 0.038 \\
\hline Nutritional status (MNA) & $21.84 \pm 2.10$ & $21.52 \pm 2.39$ & $22.17 \pm 2.19$ & 0.227 & $22.22 \pm 1.76$ & $21.41 \pm 2.06$ & 0.05 \\
\hline$\geq 1 \mathrm{ADL}$ impairment & $33(17.2)$ & $4(11.1)$ & $6(13.0)$ & 1 & $13(22.4)$ & $10(19.2)$ & 0.861 \\
\hline$\geq 1$ IADL impairment & $57(29.7)$ & $13(36.1)$ & $13(28.3)$ & 0.604 & 17 (29.3) & $14(26.9)$ & 0.948 \\
\hline Perceived Health (EQ-VAS) & $69.35 \pm 14.74$ & $70.22 \pm 12.96$ & $73.11 \pm 13.62$ & 0.336 & $68.14 \pm 16.43$ & $66.73 \pm 14.58$ & 0.639 \\
\hline Moderate Physical Activity & $78(40.8)$ & $17(47.2)$ & $23(50.0)$ & 0.978 & 19 (33.3) & $19(36.5)$ & 0.881 \\
\hline Physical activity (RAPA) & $3.35 \pm 1.61$ & $3.81 \pm 1.70$ & $3.70 \pm 1.71$ & 0.773 & $3.07 \pm 1.50$ & $3.02 \pm 1.49$ & 0.859 \\
\hline $\mathrm{MoCA}$ & $24.96 \pm 4.27$ & $25.94 \pm 3.26$ & $25.89 \pm 2.75$ & 0.936 & $24.51 \pm 5.10$ & $23.94 \pm 4.75$ & 0.551 \\
\hline \multicolumn{8}{|l|}{ Physical Function } \\
\hline Sarcopenia (SARC-F) & $1.58 \pm 1.81$ & $1.28 \pm 1.65$ & $0.83 \pm 1.22$ & 0.157 & $1.84 \pm 1.89$ & $2.18 \pm 2.03$ & 0.376 \\
\hline Sarcopenia (AWGS 2019) & $48(25.0)$ & 8 (22.2) & $14(30.4)$ & 0.561 & 15 (25.9) & $11(21.2)$ & 0.722 \\
\hline Sarcopenic obesity & $58(30.2)$ & $12(33.3)$ & $14(30.4)$ & 0.967 & $15(25.9)$ & $17(32.7)$ & 0.564 \\
\hline$\geq 5 \%$ weight loss & $19(9.9)$ & $8(22.2)$ & $5(10.9)$ & 0.275 & $4(6.9)$ & $2(3.8)$ & 0.777 \\
\hline At least 1 fall in past year & $51(26.6)$ & $7(19.4)$ & $11(23.9)$ & 0.829 & $15(25.9)$ & $18(34.6)$ & 0.428 \\
\hline Low mental vitality & 107 (56.9) & $16(44.4)$ & $18(39.1)$ & 0.796 & $40(71.4)$ & $33(66.0)$ & 0.695 \\
\hline Handgrip strength (kg) & $22.13 \pm 7.20$ & $28.05 \pm 6.20$ & $27.40 \pm 6.37$ & 0.642 & $18.72 \pm 4.73$ & $17.17 \pm 4.40$ & 0.081 \\
\hline Chair time (s) & $13.52 \pm 5.37$ & $12.55 \pm 3.88$ & $11.98 \pm 4.27$ & 0.54 & $14.09 \pm 6.38$ & $15.04 \pm 5.65$ & 0.439 \\
\hline Chair time $\geq 12 \mathrm{~s}$ & $93(52.8)$ & $18(52.9)$ & $17(38.6)$ & 0.303 & $26(51.0)$ & $32(68.1)$ & 0.13 \\
\hline Gait speed & $0.91 \pm 0.27$ & $0.96 \pm 0.27$ & $0.97 \pm 0.27$ & 0.915 & $0.90 \pm 0.29$ & $0.82 \pm 0.23$ & 0.111 \\
\hline SPPB score & $9.58 \pm 2.33$ & $10.06 \pm 1.82$ & $10.35 \pm 2.45$ & 0.552 & $9.26 \pm 2.36$ & $8.92 \pm 2.31$ & 0.453 \\
\hline \multicolumn{8}{|l|}{ Body Composition } \\
\hline ASM $\left(\mathrm{kg} / \mathrm{m}^{2}\right)$ & $7.12 \pm 1.75$ & $7.79 \pm 1.74$ & $7.82 \pm 1.80$ & 0.933 & $6.56 \pm 1.67$ & $6.67 \pm 1.43$ & 0.709 \\
\hline Normal muscle mass & $135(70.3)$ & $26(72.2)$ & $31(67.4)$ & 0.818 & 39 (67.2) & $39(75.0)$ & 0.494 \\
\hline Muscle quality $\wedge$ & 32.65 (10.49) & $33.89 \pm 11.37$ & $35.07 \pm 12.17$ & 0.659 & $32.64 \pm 10.30$ & $30.48 \pm 8.24$ & 0.233 \\
\hline Phase Angle (50Khz) & $4.98(0.99)$ & $5.04 \pm 1.03$ & $5.17 \pm 0.97$ & 0.558 & $4.96 \pm 1.13$ & $4.78 \pm 0.80$ & 0.353 \\
\hline Obese (by fat mass \%) & 119 (62.0) & $24(66.7)$ & $29(63.0)$ & 0.914 & $29(50.0)$ & $37(71.2)$ & 0.039 \\
\hline Body fat percentage (BF\%) & $33.39 \pm 8.79$ & $27.48 \pm 6.08$ & $28.34 \pm 8.30$ & 0.603 & $36.20 \pm 7.81$ & $38.81 \pm 6.98$ & 0.069 \\
\hline Fat Mass Index & $8.84 \pm 3.49$ & $6.92 \pm 2.50$ & $7.54 \pm 3.40$ & 0.363 & $9.27 \pm 3.25$ & $10.84 \pm 3.32$ & 0.014 \\
\hline Fat Free Mass Index & $16.93 \pm 2.04$ & $17.76 \pm 1.86$ & $18.19 \pm 2.03$ & 0.329 & $15.78 \pm 1.70$ & $16.51 \pm 1.67$ & 0.026 \\
\hline Fat Mass to Fat Free Mass & $0.53 \pm 0.21$ & $0.39 \pm 0.13$ & $0.42 \pm 0.19$ & 0.462 & $0.59 \pm 0.21$ & $0.65 \pm 0.19$ & 0.104 \\
\hline Visceral fat area $\left(\mathrm{cm}^{2}\right)$ & $101.80 \pm 47.32$ & $75.47 \pm 27.88$ & $88.43 \pm 40.09$ & 0.117 & $109.26 \pm 52.54$ & $124.89 \pm 46.99$ & 0.134 \\
\hline Visceral fat area $>100 \mathrm{~cm}^{2}$ & $78(46.2)$ & $4(12.1)$ & $16(37.2)$ & 0.028 & $24(53.3)$ & $34(70.8)$ & 0.127 \\
\hline Body cell mass & $25.69 \pm 7.25$ & $29.15 \pm 8.28$ & $30.16 \pm 6.14$ & 0.527 & $21.69 \pm 5.74$ & $23.73 \pm 5.56$ & 0.063 \\
\hline
\end{tabular}

$n(\%) ;$ mean $\pm S D$; significant in bold; sarcopenic obesity definition: Male: ASM: BMI < 0.789 \& BF\% > 25; Female: ASM : BMI<0.512 \& BF\%>35.

AWGS, Asian Workgroup for Sarcopenia; BMI, Body Mass Index; ^HGS/ASMBMI; MNA, mini nutritional assessment; Muscle quality ^:HGS/ASMBMI.

the non-diabetic population. Bioimpedance analysis revealed significantly lower mean ASM $(\mathrm{kg} / \mathrm{m} 2)$ mass, FFMI and body cell mass, and higher BF\%, FMI, FM/FFM and VFA in females compared to males, in both the DM and non-diabetic population. Mean whole body phase angle was significantly lower in females with DM compared to males $(4.78 \pm 0.80$ versus $5.17 \pm 0.97$. Muscle quality was significantly lower in females with DM, $30.48 \pm 8.24$ compared with males $35.07 \pm$ 12.17. Body cell mass was lower in females regardless of the DM status. 
TABLE 2 | Characteristics table displaying between gender differences by Diabetes Mellitus (DM) Status.

\begin{tabular}{|c|c|c|c|c|c|c|c|}
\hline & \multirow[t]{2}{*}{ All $(n=192)$} & \multicolumn{3}{|c|}{$\begin{array}{c}\text { No DM } \\
94(49.0 \%)\end{array}$} & \multicolumn{3}{|c|}{$\begin{array}{c}\text { DM } \\
98(51.0 \%)\end{array}$} \\
\hline & & $\begin{array}{c}\text { Male } \\
36(38.3 \%)\end{array}$ & $\begin{array}{c}\text { Female } \\
58(61.7 \%)\end{array}$ & $P$ value & $\begin{array}{c}\text { Male } \\
46(46.9 \%)\end{array}$ & $\begin{array}{c}\text { Female } \\
52(53.1 \%)\end{array}$ & $P$ value \\
\hline \multicolumn{8}{|l|}{ Demographics } \\
\hline Age & $73.04 \pm 5.73$ & $74.39 \pm 6.74$ & $72.52 \pm 5.50$ & 0.145 & $72.59 \pm 5.41$ & $73.08 \pm 5.52$ & 0.659 \\
\hline Ethnicity & & & & 0.871 & & & 0.287 \\
\hline Chinese & 159 (82.8) & 32 (88.9) & $50(86.2)$ & & $33(71.7)$ & $44(84.6)$ & \\
\hline Malay & $13(6.8)$ & $2(5.6)$ & $4(6.9)$ & & $4(8.7)$ & $3(5.8)$ & \\
\hline Indian & 19 (9.9) & $2(5.6)$ & $3(5.2)$ & & 9 (19.6) & $5(9.6)$ & \\
\hline Others & $1(0.5)$ & $0(0.0)$ & $1(1.7)$ & & & & \\
\hline $\mathrm{BMI}\left(\mathrm{kg} / \mathrm{m}^{2}\right)$ & $25.89 \pm 4.17$ & $24.69 \pm 3.52$ & $25.24 \pm 3.76$ & 0.483 & $26.00 \pm 4.57$ & $27.33 \pm 4.33$ & 0.143 \\
\hline BMI status & & & & 0.68 & & & 0.083 \\
\hline Normal & $50(26.0)$ & $13(36.1)$ & $16(27.6)$ & & $11(23.9)$ & $10(19.2)$ & \\
\hline Overweight & $61(31.8)$ & $8(22.2)$ & $14(24.1)$ & & $13(28.3)$ & $26(50.0)$ & \\
\hline Obese & $81(42.2)$ & $15(41.7)$ & $28(48.3)$ & & $22(47.8)$ & $16(30.8)$ & \\
\hline Depression & $59(31.4)$ & $9(25.0)$ & $23(41.1)$ & 0.175 & $8(17.4)$ & 19 (38.0) & 0.044 \\
\hline Hyperlipidaemia & $157(81.8)$ & 29 (80.6) & $38(65.5)$ & 0.183 & $42(91.3)$ & 48 (92.3) & 1 \\
\hline Hypertension & $139(72.8)$ & 29 (80.6) & $33(56.9)$ & 0.033 & $37(80.4)$ & $40(78.4)$ & 1 \\
\hline Multimorbidity & 161 (83.9) & 29 (80.6) & $36(62.1)$ & 0.098 & 45 (97.8) & $51(98.1)$ & 1 \\
\hline Education (years) & $7.84 \pm 4.27$ & $8.26 \pm 4.28$ & $7.58 \pm 4.48$ & 0.475 & $9.39 \pm 3.88$ & $6.40 \pm 3.93$ & $<0.001$ \\
\hline Calf circumference (cm) & $35.85 \pm 4.51$ & $36.17 \pm 3.55$ & $34.93 \pm 3.69$ & 0.119 & $36.44 \pm 3.12$ & $36.17 \pm 6.58$ & 0.805 \\
\hline Waist circumference (cm) & $93.05 \pm 10.93$ & $92.84 \pm 9.32$ & $89.83 \pm 12.16$ & 0.312 & $94.93 \pm 9.75$ & $96.18 \pm 10.74$ & 0.666 \\
\hline Nutritional status (MNA) & $21.84 \pm 2.10$ & $21.52 \pm 2.39$ & $22.22 \pm 1.76$ & 0.145 & $22.17 \pm 2.19$ & $21.41 \pm 2.06$ & 0.099 \\
\hline$\geq 1 \mathrm{ADL}$ impairment & $33(17.2)$ & $4(11.1)$ & $13(22.4)$ & 0.268 & $6(13.0)$ & $10(19.2)$ & 0.58 \\
\hline$\geq 1$ IADL impairment & $57(29.7)$ & $13(36.1)$ & 17 (29.3) & 0.646 & $13(28.3)$ & 14 (26.9) & 1 \\
\hline Perceived Health (EQ-VAS) & $69.35 \pm 14.74$ & $70.22 \pm 12.96$ & $68.14 \pm 16.43$ & 0.523 & $73.11 \pm 13.62$ & $66.73 \pm 14.58$ & 0.03 \\
\hline Moderate Physical Activity & $78(40.8)$ & $17(47.2)$ & 19 (33.3) & 0.262 & $23(50.0)$ & $19(36.5)$ & 0.255 \\
\hline Physical activity (RAPA) & $3.35 \pm 1.61$ & $3.81 \pm 1.70$ & $3.07 \pm 1.50$ & 0.031 & $3.70 \pm 1.71$ & $3.02 \pm 1.49$ & 0.039 \\
\hline MoCA & $24.96 \pm 4.27$ & $25.94 \pm 3.26$ & $24.51 \pm 5.10$ & 0.136 & $25.89 \pm 2.75$ & $23.94 \pm 4.75$ & 0.016 \\
\hline \multicolumn{8}{|l|}{ Physical Function } \\
\hline Sarcopenia (SARC-F) & $1.58 \pm 1.81$ & $1.28 \pm 1.65$ & $1.84 \pm 1.89$ & 0.142 & $0.83 \pm 1.22$ & $2.18 \pm 2.03$ & $<0.001$ \\
\hline Sarcopenia (AWGS 2019) & $48(25.0)$ & 8 (22.2) & 15 (25.9) & 0.879 & $14(30.4)$ & $11(21.2)$ & 0.412 \\
\hline Sarcopenic obesity & $58(30.2)$ & $12(33.3)$ & $15(25.9)$ & 0.587 & $14(30.4)$ & $17(32.7)$ & 0.982 \\
\hline$\geq 5 \%$ weight loss & 19 (9.9) & $8(22.2)$ & $4(6.9)$ & 0.065 & $5(10.9)$ & $2(3.8)$ & 0.34 \\
\hline At least 1 fall in past year & $51(26.6)$ & $7(19.4)$ & $15(25.9)$ & 0.643 & $11(23.9)$ & $18(34.6)$ & 0.349 \\
\hline Low mental vitality & 107 (56.9) & $16(44.4)$ & $40(71.4)$ & 0.018 & $18(39.1)$ & $33(66.0)$ & 0.015 \\
\hline Handgrip strength (kg) & $22.13 \pm 7.20$ & $28.05 \pm 6.20$ & $18.72 \pm 4.73$ & $<0.001$ & $27.40 \pm 6.37$ & $17.17 \pm 4.40$ & $<0.001$ \\
\hline Chair time (s) & $13.52 \pm 5.37$ & $12.55 \pm 3.88$ & $14.09 \pm 6.38$ & 0.214 & $11.98 \pm 4.27$ & $15.04 \pm 5.65$ & 0.005 \\
\hline Chair time $\geq 12 \mathrm{~s}$ & 93 (52.8) & $18(52.9)$ & $26(51.0)$ & 1 & $17(38.6)$ & $32(68.1)$ & 0.009 \\
\hline Gait speed & $0.91 \pm 0.27$ & $0.96 \pm 0.27$ & $0.90 \pm 0.29$ & 0.297 & $0.97 \pm 0.27$ & $0.82 \pm 0.23$ & 0.003 \\
\hline SPPB score & $9.58 \pm 2.33$ & $10.06 \pm 1.82$ & $9.26 \pm 2.36$ & 0.087 & $10.35 \pm 2.45$ & $8.92 \pm 2.31$ & 0.004 \\
\hline \multicolumn{8}{|l|}{ Body Composition } \\
\hline $\operatorname{ASM}\left(\mathrm{kg} / \mathrm{m}^{2}\right)$ & $7.12 \pm 1.75$ & $7.79 \pm 1.74$ & $6.56 \pm 1.67$ & 0.001 & $7.82 \pm 1.80$ & $6.67 \pm 1.43$ & 0.001 \\
\hline Normal muscle mass & 135 (70.3) & $26(72.2)$ & $39(67.2)$ & 0.781 & $31(67.4)$ & $39(75.0)$ & 0.543 \\
\hline Muscle quality ^ & $32.65(10.49)$ & $33.89 \pm 11.37$ & $32.64 \pm 10.30$ & 0.587 & $35.07 \pm 12.17$ & $30.48 \pm 8.24$ & 0.031 \\
\hline Phase Angle (50Khz) & 4.98 (0.99) & $5.04 \pm 1.03$ & $4.96 \pm 1.13$ & 0.716 & $5.17 \pm 0.97$ & $4.78 \pm 0.80$ & 0.031 \\
\hline Obese (by fat mass \%) & $119(62.0)$ & $24(66.7)$ & $29(50.0)$ & 0.171 & $29(63.0)$ & $37(71.2)$ & 0.523 \\
\hline Body fat $(\%)$ & $33.39 \pm 8.79$ & $27.48 \pm 6.08$ & $36.20 \pm 7.81$ & $<0.001$ & $28.34 \pm 8.30$ & $38.81 \pm 6.98$ & $<0.001$ \\
\hline Fat Mass Index & $8.84 \pm 3.49$ & $6.92 \pm 2.50$ & $9.27 \pm 3.25$ & $<0.001$ & $7.54 \pm 3.40$ & $10.84 \pm 3.32$ & $<0.001$ \\
\hline Fat Free Mass Index & $16.93 \pm 2.04$ & $17.76 \pm 1.86$ & $15.78 \pm 1.70$ & $<0.001$ & $18.19 \pm 2.03$ & $16.51 \pm 1.67$ & $<0.001$ \\
\hline Fat Mass to Fat Free Mass & $0.53 \pm 0.21$ & $0.39 \pm 0.13$ & $0.59 \pm 0.21$ & $<0.001$ & $0.42 \pm 0.19$ & $0.65 \pm 0.19$ & $<0.001$ \\
\hline Visceral fat area $\left(\mathrm{cm}^{2}\right)$ & $101.80 \pm 47.32$ & $75.47 \pm 27.88$ & $109.26 \pm 52.54$ & 0.001 & $88.43 \pm 40.09$ & $124.89 \pm 46.99$ & $<0.001$ \\
\hline Visceral fat area $>100 \mathrm{~cm}^{2}$ & $78(46.2)$ & $4(12.1)$ & $24(53.3)$ & $<0.001$ & $16(37.2)$ & $34(70.8)$ & 0.003 \\
\hline Body cell mass & $25.69 \pm 7.25$ & $29.15 \pm 8.28$ & $21.69 \pm 5.74$ & $<0.001$ & $30.16 \pm 6.14$ & $23.73 \pm 5.56$ & $<0.001$ \\
\hline
\end{tabular}

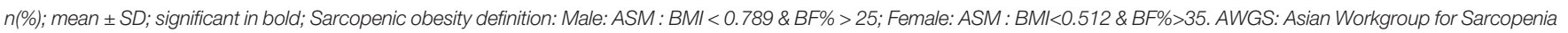
BMI, Body Mass Index; ^HGS/ASMBMI; MNA, mini nutritional assessment; Muscle quality ^:HGS/ASMBMI.

The association of sarcopenic obesity, obesity, sarcopenia, and body composition to DM status are displayed in Table 3. Both unadjusted and adjusted models for BMI were significantly associated to DM status, with odds ratios of $1.11(95 \%$ CI 1.02 -
1.21); $\mathrm{p}=0.013$ after adjustment for weight loss, physical activity, gender, ethnicity, age, education, EQ-VAS, GDS, mental vitality, physical performance, and MoCA. WC was significantly associated with DM status (odds ratio 1.04 (95\% CI 1.00-1.08; 
TABLE 3 | Sarcopenic obesity, obesity, sarcopenia definitions and body composition analysis associations with Diabetes Mellitus status.

\begin{tabular}{|c|c|c|c|c|c|}
\hline Variables & Model type & OR (97.5\% Cl) & Specificity & Sensitivity & AUC \\
\hline \multirow[t]{2}{*}{$\mathrm{BMl}$} & Adjusted & $1.11(1.02-1.21) ; \mathrm{p}=0.013$ & 0.71 & 0.56 & 0.66 \\
\hline & Unadjusted & $1.11(1.03-1.19) ; P=0.007$ & 0.66 & 0.61 & 0.61 \\
\hline \multirow[t]{2}{*}{$\mathrm{BMI} \geq 27.5$} & Adjusted & $2.27(0.99-5.33) ; p=0.05$ & 0.70 & 0.60 & 0.66 \\
\hline & Unadjusted & 2.45(1.15-5.34); $P=0.02$ & 0.76 & 0.40 & 0.59 \\
\hline \multirow[t]{2}{*}{ Waist Circumference (cm) } & Adjusted & $1.03(0.99-1.07) ; p=0.15$ & 0.68 & 0.69 & 0.70 \\
\hline & Unadjusted & $1.04(1.00-1.08) ; P=0.03$ & 0.77 & 0.53 & 0.63 \\
\hline \multirow[t]{2}{*}{ Visceral Fat Area $\mathrm{cm}^{2}$} & Adjusted & $1.01(0.99-1.01) ; p=0.07$ & 0.62 & 0.71 & 0.66 \\
\hline & Unadjusted & $1.00(0.99-1.01) ; P=0.08$ & 0.64 & 0.58 & 0.58 \\
\hline \multirow{2}{*}{ Visceral fat area $>100 \mathrm{~cm}^{2}$} & Adjusted & $2.94(1.41-6.38) ; p=0.005$ & 0.69 & 0.76 & 0.54 \\
\hline & Unadjusted & 2.17(1.17-4.08); $P=0.01$ & 0.64 & 0.55 & 0.59 \\
\hline \multirow[t]{2}{*}{ Percentage Body Fat (BF\%) } & Adjusted & $1.02(0.98-1.07) ; p=0.22$ & 0.79 & 0.41 & 0.62 \\
\hline & Unadjusted & $1.01(0.98-1.05) ; P=0.44$ & 0.77 & 0.42 & 0.54 \\
\hline \multirow[t]{2}{*}{ Obese (BF\% by gender)* } & Adjusted & $1.36(0.72-2.57) ; p=0.33$ & 0.59 & 0.64 & 0.62 \\
\hline & Unadjusted & $1.55(0.87-2.82) ; P=0.14$ & 0.43 & 0.67 & 0.55 \\
\hline \multirow[t]{2}{*}{ Fat Mass Index } & Adjusted & $1.11(1.00-1.23) ; p=0.04$ & 0.88 & 0.39 & 0.65 \\
\hline & Unadjusted & $1.07(0.99-1.18) ; P=0.07$ & 0.74 & 0.44 & 0.58 \\
\hline \multirow[t]{2}{*}{ Fat Free Mass Index } & Adjusted & $1.23(1.02-1.49) ; p=0.03$ & 0.78 & 0.51 & 0.66 \\
\hline & Unadjusted & $1.21(1.05-1.42) ; P=0.01$ & 0.42 & 0.81 & 0.61 \\
\hline \multirow[t]{2}{*}{ Fat Mass to Fat Free Mass } & Adjusted & $2.94(0.55-17.00) ; P=0.21$ & 0.84 & 0.37 & 0.63 \\
\hline & Unadjusted & $1.81(0.48-7.01) ; P=0.39$ & 0.77 & 0.42 & 0.54 \\
\hline \multirow[t]{2}{*}{ Appendicular Skeletal Muscle Mass Index (kg/m2) } & Adjusted & $1.02(0.84-1.25) ; p=0.85$ & 0.44 & 0.77 & 0.62 \\
\hline & Unadjusted & 1.05(0.90-1.26); P=0.50 & 0.37 & 0.83 & 0.57 \\
\hline \multirow[t]{2}{*}{ Muscle Quality } & Adjusted & $0.99(0.96-1.03) \quad ; p=0.75$ & 0.50 & 0.73 & 0.62 \\
\hline & Unadjusted & 0.99(0.97-1.02); P=0.76 & 0.17 & 0.89 & 0.51 \\
\hline \multirow[t]{2}{*}{ Sarcopenia^ } & Adjusted & $0.93(0.46-1.88) ; p=0.84$ & 0.47 & 0.73 & 0.62 \\
\hline & Unadjusted & $1.04(0.54-2.01) ; P=0.90$ & 0.75 & 0.26 & 0.50 \\
\hline \multirow[t]{2}{*}{ Sarcopenic Obesity ${ }^{\S}$} & Adjusted & $0.91(0.45-1.87) ; p=0.81$ & 0.49 & 0.73 & 0.61 \\
\hline & Unadjusted & $1.13(0.61-2.11) ; P=0.70$ & 0.71 & 0.32 & 0.51 \\
\hline
\end{tabular}

Adjustment for weight loss, physical activity, gender, ethnicity, age, education, EQ-VAS, GDS, mental vitality, physical performance, MoCA; Specificity and Sensitivity at Youden point, AUC, Area under receiver operating characteristic curve; significant models in bold. BMI, body mass index.

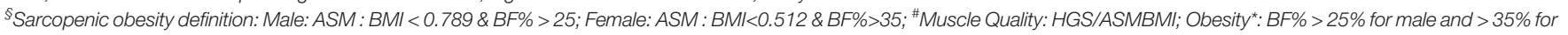
female; sarcopenia^: 2019 Asian Workgroup for Sarcopenia definition.

$\mathrm{p}=0.03$ ) but loses significance after adjustment for the variables above. Both unadjusted and adjusted models for FFMI were significantly associated with DM status (odds ratios of 1.21; 95\% CI 1.05-1.42; $\mathrm{P}=0.01$ and 1.23 (95\% CI 1.02-1.49; $\mathrm{p}=0.03$ respectively). Adjusted FMI was significantly associated with DM status (odds ratio of 1.11; 95\% CI 1.00-1.23; $\mathrm{p}=0.04$ ).

\section{DISCUSSION}

Our study highlights two very significant aspects. Firstly, there were insignificant physical function, cognition, and body composition differences within gender for pre-frail male and female except for higher prevalence of obesity defined by BMI and BF\%, increase FMI and FFMI in females with DM. Secondly, there were significant differences between gender for those with DM where females overall had lower education levels, lower perceived health, lower RAPA scores, higher prevalence of depression and low mental vitality, lower overall physical function as shown by low SPPB scores, low GS and HGS, lower MoCA, lower muscle mass, lower muscle quality, body cell mass and whole body phase angle with higher FMI, FM/FFM and VFA. A trend was observed for female with $\mathrm{DM}$ to have lower mean MNA scores compared to male. Overall obesity defined by BMI, FFMI, FMI and VFA
$>100 \mathrm{~cm}^{2}$ was significantly associated with DM status in prefrail older adults, and not BF\%.

Chronic inflammation is a key element in pre-frailty, sarcopenia, sarcopenic obesity and metabolic diseases such as diabetes (42-44). Body composition changes with ageing especially increase in visceral fat and ectopic fat distribution is pro-inflammatory and obesity is associated among others with high baseline C-reactive protein and interleukin-6 (45). Intramuscular fat infiltration together with sedentary lifestyle, poor dietary habits and effects of medications further aggravates insulin resistance in older adults (46). Obesity is associated with mitochondrial dysfunction, which in is also thought to be responsible for insulin resistance, frailty, and sarcopenia (4749). Almost 8 out of 10 of females and 7 in 10 males in our study were classified as overweight or obese based on BMI cut-off. In recent years, there has been increasing literature on metabolically healthy obesity (MHO) and metabolically unhealthy obesity (MUO). Increase in FFMI in older obese women has been shown to be an independently associated with MUO which in turn is a predictor of adverse metabolic health (50). Pre-frail females with DM in our study had significantly higher FFMI, FMI, hypertension and hyperlipidaemia prevalence compared with non-diabetics. After adjustment, BMI, FFMI, FMI and VFA $>100 \mathrm{~cm}^{2}$ were independently associated with DM status further supporting the concept of MUO. 
In our study, there were no functional, cognition and body composition differences within gender except for increase in BMI, FMI, FFMI and BF\% in females. While muscle quality was overall lower in females, there was no significant differences in those with DM and no DM. There were significant differences in function, cognition, perceived health, depression and body composition between gender in those with DM. Hormonal changes with ageing and menopause such as a rapid fall in oestrogen levels, higher testosterone levels and lower sex hormone-binding globulin are associated with increase in body weight, redistribution of FM, decrease in FFM, insulin resistance and Type $2 \mathrm{DM}$ in women compared to men (51). Within gender, there was a trend for diabetics to have higher ASM $(\mathrm{kg} /$ $\mathrm{m}^{2}$ ) and FFMI but significant only for FFMI in females without any significant impact on functional status.

Phase angle is considered as an index of muscle quality and as an indicator of muscle catabolism. It is also a measure of cell vitality and a prognostic marker for frailty, mortality, and functional status (52). Similarly, body cell mass is a measure of metabolic activity, indicator of loss of muscle mass with ageing, nutrition, and inflammation (53). While there was no significant within gender differences for phase angle between diabetics and non-diabetics, pre-frail female with DM exhibited a significantly lower phase angle compared with males. This was further supported by overall lower muscle quality in females with DM. This is in contrary to a recently published study where males with DM had overall lower phase angle (52). Body cell mass was significantly lower in females regardless of their diabetes status which requires further validation.

Diabetes has been associated with low muscle quality, muscle mass and strength which was not shown in our pre-frail participants (54). While the possible explanations include the shared pathophysiology including chronic inflammation, hormonal changes with age and dysfunctional adipose tissue, the effect of medications like metformin was not evaluated. Metformin has immunomodulatory effect and has been associated with lower risk of frailty, lower mortality, lower inflammation in diabetics and protective against sarcopenia $(44,55,56)$. While we have no information on metformin use in our study participants, metformin use in patients with DM in a local tertiary centre in 2017 was 59.6\%. Similarly, while sarcopenic obesity and DM are closely associated due to shared risk factors, this was not shown in our pre-frail participants (57).

\section{Methodological Considerations}

Our study has several strengths. It represents a snapshot of community dwelling pre-frail older adults in Singapore and to date, there is limited data on pre-frail body composition especially comparing diabetics and non-diabetics. The study utilizers objective measures of muscle strength and robust measures of body composition, together with validated clinical tools to measure physical performance, cognitive function, and perceived health. Our study has several limitations which warrants mention. Absolute consensus for an operational definition for prefrailty has not been achieved, however it is acknowledged in a recent publication that it lies along the frailty continuum (58). However, we utilized a well validated FRAIL score to define the cohort. Due to the cross-sectional study design, lack of information on DM control, duration and treatment, the findings cannot be generalized and needs to be validated in longitudinal studies. We are unable to elucidate the magnitude of the bidirectional relationship of prefrail and DM, and effect of DM with or without treatment on the trajectory of body composition. We did not have information on metformin use in pre-frail diabetics and if it conferred a protective role. The DM status is self-reported which may be subject to recall bias. Our study included only pre-frail participants; thus, our findings cannot be extrapolated to robust or frail populations. Despite the small sample size, the prevalence of self-reported DM was $51 \%$ and our study has generated interesting findings which requires further validation. Lastly, body composition measured by BIA may be affected by hydration, oedema and fasting but as our participants were community dwelling older adults, most of our participants were generally well and there was no fasting involved.

\section{Study Highlights and Future Directions}

Our study further adds to the evolving literature on crossroad between frailty and diabetes where within gender, pre-frail older adults had similar body composition, cognition, and functional status. These findings should be explored in an external cohort for further validation. Both frailty and diabetes share a bi-directional relationship where frailty accelerates diabetes development, and diabetes accelerates frailty and ageing (22). Frailty is reversible, and predisposing factors for DM are modifiable with exercise and multidomain interventions $(17,59)$. While metformin has shown promising effect in extending health span, down regulating inflammation in diabetics and, its effects on delaying and reversing frailty and changing the trajectory of DM in pre-frail older adults is still an area of ongoing research (44). Longitudinal studies are needed to understand the magnitude and impact of chronic inflammation in both pre-frail older adults with and without DM, trajectory of DM development in non-diabetic pre-frail and progression of frailty and disability in pre-frail diabetics, and the role of metformin (55).

\section{CONCLUSIONS}

There were insignificant physical function, cognition, and body composition differences within gender for pre-frail male and female except for higher prevalence of obesity defined by BMI and BF\%, increase FMI and FFMI in females with DM. There were significant differences between gender for those with DM where females had overall lower muscle quality, lower physical function, cognition, perceived health with higher prevalence of depression. BMI, VFA $>100$, FMI and FFMI were found to be independently associated with DM status in pre-frail older adults. Further longitudinal studies are required to understand the pathogenesis, trajectory of DM and protective role of oral hypoglycemics in pre-frail older adults.

\section{DATA AVAILABILITY STATEMENT}

The raw data supporting the conclusions of this article will be made available by the authors, without undue reservation. 


\section{ETHICS STATEMENT}

The studies involving human participants were reviewed and approved by The National Healthcare Group Domain Specific Review Board (Reference: 2017/00035 and 2018/01183). The patients/participants provided their written informed consent to participate in this study.

\section{AUTHOR CONTRIBUTIONS}

RM: funding acquisition. RM and JM: methodology and conceptualization. RM and JS: formal analysis. RM: methodology and project administration. RM and JS: writingoriginal draft. RM, JS, and JM - review and editing. All authors contributed to the article and approved the submitted version.

\section{REFERENCES}

1. United Nations. World Population Ageing 2019 (2019). Available at: https:// www.un.org/en/development/desa/population/publications/pdf/ageing/ WorldPopulationAgeing2019-Highlights.pdf.

2. Baumgartner RN. Body Composition in Healthy Aging. Ann N Y Acad Sci (2000) 904:437-48. doi: 10.1111/j.1749-6632.2000.tb06498.x

3. Ponti F, Santoro A, Mercatelli D, Gasperini C, Conte M, Martucci M, et al. Aging and Imaging Assessment of Body Composition: From Fat to Facts. Front Endocrinol (Lausanne) (2019) 10:861. doi: 10.3389/fendo.2019.00861

4. Lu Y, Shu H, Zheng Y, Li C, Liu M, Chen Z, et al. Comparison of Fat-Free Mass Index and Fat Mass Index in Chinese Adults. Eur J Clin Nutr (2012) 66 (9):1004-7. doi: 10.1038/ejcn.2012.86

5. Ponti F, Santoro A, Mercatelli D, Gasperini C, Conte M, Martucci M, et al. Aging and Imaging Assessment of Body Composition: From Fat to Facts. Front Endocrinol (2020) 10:861. doi: 10.3389/fendo.2019.00861

6. Sinclair A, Saeedi P, Kaundal A, Karuranga S, Malanda B, Williams R. Diabetes and Global Ageing Among 65-99-Year-Old Adults: Findings From the International Diabetes Federation Diabetes Atlas, 9(Th) Edition. Diabetes Res Clin Pract (2020) 162:108078. doi: 10.1016/j.diabres.2020.108078

7. Merchant RA, Chen MZ, Tan LWL, Lim MY, Ho HK, van Dam RM. Singapore Healthy Older People Everyday (HOPE) Study: Prevalence of Frailty and Associated Factors in Older Adults. J Am Med Dir Assoc (2017) 18(8):734.e9-14. doi: 10.1016/j.jamda.2017.04.020

8. Chang AM, Halter JB. Aging and Insulin Secretion. Am J Physiol Endocrinol Metab (2003) 284(1):E7-12. doi: 10.1152/ajpendo.00366.2002

9. Kalyani RR, Corriere M, Ferrucci L. Age-Related and Disease-Related Muscle Loss: The Effect of Diabetes, Obesity, and Other Diseases. Lancet Diabetes Endocrinol (2014) 2(10):819-29. doi: 10.1016/S2213-8587(14)70034-8

10. Onwudiwe NC, Stuart B, Zuckerman IH, Sorkin JD. Obesity and Medicare Expenditure: Accounting for Age-Related Height Loss. Obesity (Silver Spring) (2011) 19(1):204-11. doi: 10.1038/oby.2010.145

11. Mainous AG3rd, Tanner RJ, Jo A, Anton SD. Prevalence of Prediabetes and Abdominal Obesity Among Healthy-Weight Adults: 18-Year Trend. Ann Fam Med (2016) 14(4):304-10. doi: 10.1370/afm.1946

12. Jo A, Mainous AGIII. Informational Value of Percent Body Fat With Body Mass Index for the Risk of Abnormal Blood Glucose: A Nationally Representative Cross-Sectional Study. BMJ Open (2018) 8(4):e019200. doi: 10.1136/bmjopen-2017-019200

13. Hou Q, Guan Y, Yu W, Liu X, Wu L, Xiao M, et al. Associations Between Obesity and Cognitive Impairment in the Chinese Elderly: An Observational Study. Clin Interv Aging (2019) 14:367-73. doi: 10.2147/CIA.S192050

14. Merchant RA, Wong MWK, Lim JY, Morley JE. Association of Central Obesity and High Body Mass Index With Function and Cognition in Older Adults. Endocr Connect (2021) 10(8):909-17. doi: 10.1530/EC-21-0223

15. Chen Y, He D, Yang T, Zhou H, Xiang S, Shen L, et al. Relationship Between Body Composition Indicators and Risk of Type 2 Diabetes Mellitus in Chinese

\section{FUNDING}

This study is part of a larger project that has been funded by Ministry of Health of Singapore: Healthy Ageing Innovation Grant under National Innovation Challenge on Active and Confident Ageing (Award No: $\mathrm{MOH} / \mathrm{NIC} /$ HAIG02/2017) and National Medical Research Council (HSRG-HP17Jun003).

\section{ACKNOWLEDGMENTS}

The authors wish to thank Bukit Batok and Chua Chu Kang National University Polyclinic, Singapore for facilitating participant recruitment.

Adults. BMC Public Health (2020) 20(1):452. doi: 10.1186/s12889-020$08552-5$

16. Kim CH, Kim HK, Kim EH, Bae SJ, Park JY. Association Between Changes in Body Composition and Risk of Developing Type 2 Diabetes in Koreans. Diabetes Med (2014) 31(11):1393-8. doi: 10.1111/dme.12527

17. Dent E, Morley JE, Cruz-Jentoft AJ, Woodhouse L, Rodriguez-Manas L, Fried LP, et al. Physical Frailty: ICFSR International Clinical Practice Guidelines for Identification and Management. J Nutr Health Aging (2019) 23(9):771-87. doi: 10.1007/s12603-019-1273-z

18. Dent E, Morley JE, Cruz-Jentoft AJ, Arai H, Kritchevsky SB, Guralnik J, et al. International Clinical Practice Guidelines for Sarcopenia (ICFSR): Screening, Diagnosis and Management. J Nutr Health Aging (2018) 22(10):1148-61. doi: 10.1007/s12603-018-1139-9

19. Castro-Rodríguez M, Carnicero JA, Garcia-Garcia FJ, Walter S, Morley JE, Rodríguez-Artalejo F, et al. Frailty as a Major Factor in the Increased Risk of Death and Disability in Older People With Diabetes. J Am Med Dir Assoc (2016) 17(10):949-55. doi: 10.1016/j.jamda.2016.07.013

20. Yoon S-J, Kim K-I. Frailty and Disability in Diabetes. Ann Geriatr Med Res (2019) 23(4):165-9. doi: 10.4235/agmr.19.0036

21. Chen MZ, Wong MWK, Lim JY, Merchant RA. Frailty and Quality of Life in Older Adults With Metabolic Syndrome - Findings From the Healthy Older People Everyday (HOPE) Study. J Nutr Health Aging (2021) 25(5):637-44. doi: 10.1007/s12603-021-1609-3

22. Perkisas S, Vandewoude M. Where Frailty Meets Diabetes. Diabetes Metab Res Rev (2016) 32(S1):261-7. doi: 10.1002/dmrr.2743

23. Veronese N, Stubbs B, Fontana L, Trevisan C, Bolzetta F, De Rui M, et al. Frailty Is Associated With an Increased Risk of Incident Type 2 Diabetes in the Elderly. J Am Med Dir Assoc (2016) 17(10):902-7. doi: 10.1016/ j.jamda.2016.04.021

24. Strain WD, Down S, Brown P, Puttanna A, Sinclair A. Diabetes and Frailty: An Expert Consensus Statement on the Management of Older Adults With Type 2 Diabetes. Diabetes Ther (2021) 12(5):1227-47. doi: 10.1007/s13300021-01035-9

25. O'Caoimh R, Sezgin D, O'Donovan MR, Molloy DW, Clegg A, Rockwood K, et al. Prevalence of Frailty in 62 Countries Across the World: A Systematic Review and Meta-Analysis of Population-Level Studies. Age Ageing (2020) 50 (1):96-104. doi: 10.1093/ageing/afaa219

26. Park SW, Goodpaster BH, Lee JS, Kuller LH, Boudreau R, de Rekeneire $\mathrm{N}$, et al. Excessive Loss of Skeletal Muscle Mass in Older Adults With Type 2 Diabetes. Diabetes Care (2009) 32(11):1993-7. doi: 10.2337/dc090264

27. Lim S-L, Tong C-Y, Ang E, Lee EJ-C, Loke W-C, Chen Y, et al. Development and Validation of 3-Minute Nutrition Screening (3MinNS) Tool for Acute Hospital Patients in Singapore. Asia Pac J Clin Nutr (2009) 18(3):395-403.

28. Katz S, Ford AB, Moskowitz RW, Jackson BA, Jaffe MW. Studies of Illness in the Aged the Index of Adl: a Standardized Measure of Biological and 
Psychosocial Function. JAMA (1963) 185:914-9. doi: 10.1001/jama.1963. 03060120024016

29. Lawton MP, Brody EM. Assessment of Older People: Self-Maintaining and Instrumental Activities of Daily Living. Gerontologist (1969) 9(3):179-86. doi: 10.1093/geront/9.3_Part_1.179

30. Morley JE, Malmstrom TK, Miller DK. A Simple Frailty Questionnaire (FRAIL) Predicts Outcomes in Middle Aged African Americans. J Nutr Health Aging (2012) 16(7):601-8. doi: 10.1007/s12603-012-0084-2

31. Malmstrom TK, Morley JE. SARC-F: A Simple Questionnaire to Rapidly Diagnose Sarcopenia. J Am Med Dir Assoc (2013) 14(8):531-2. doi: 10.1016/ j.jamda.2013.05.018

32. Dong Y, Lee WY, Basri NA, Collinson SL, Merchant RA, Venketasubramanian $\mathrm{N}$, et al. The Montreal Cognitive Assessment is Superior to the Mini-Mental State Examination in Detecting Patients at Higher Risk of Dementia. Int Psychogeriatr (2012) 24(11):1749-55. doi: 10.1017/S1041610212001068

33. Topolski TD, LoGerfo J, Patrick DL, Williams B, Walwick J, Patrick MB. The Rapid Assessment of Physical Activity (RAPA) Among Older Adults. Prev Chronic Dis (2006) 3(4):A118.

34. Yang YJ. An Overview of Current Physical Activity Recommendations in Primary Care. Korean J Fam Med (2019) 40(3):135-42. doi: 10.4082/kjfm.19.0038

35. Dias FL, Teixeira AL, Guimarães HC, Barbosa MT, Resende EPF, Beato RG, et al. Accuracy of the 15-Item Geriatric Depression Scale (GDS-15) in a Community-Dwelling Oldest-Old Sample: The Pietà Study. Trends Psychiatry Psychother (2017) 39(4):276-9. doi: 10.1590/2237-6089-2017-0046

36. Masciocchi E, Maltais M, El Haddad K, Virecoulon Giudici K, Rolland Y, Vellas B, et al. Defining Vitality Using Physical and Mental Well-Being Measures in Nursing Homes: A Prospective Study. J Nutr Health Aging (2020) 24(1):37-42. doi: 10.1007/s12603-019-1285-8

37. de Boer AGEM, van Lanschot JJB, Stalmeier PFM, van Sandick JW, Hulscher JBF, de Haes JCJM, et al. Is a Single-Item Visual Analogue Scale as Valid, Reliable and Responsive as Multi-Item Scales in Measuring Quality of Life? Qual Life Res (2004) 13(2):311-20. doi: 10.1023/B:QURE.0000018499.64574.1f

38. WHO Expert Consultation. Appropriate Body-Mass Index for Asian Populations and Its Implications for Policy and Intervention Strategies. Lancet (2004) 363(9403):157-63. doi: 10.1016/S0140-6736(03)15268-3

39. Batsis JA, Mackenzie TA, Lopez-Jimenez F, Bartels SJ. Sarcopenia, Sarcopenic Obesity, and Functional Impairments in Older Adults: National Health and Nutrition Examination Surveys 1999-2004. Nutr Res (2015) 35(12):1031-9. doi: 10.1016/j.nutres.2015.09.003

40. Chen LK, Woo J, Assantachai P, Auyeung TW, Chou MY, Iijima K, et al. Asian Working Group for Sarcopenia: 2019 Consensus Update on Sarcopenia Diagnosis and Treatment. J Am Med Dir Assoc (2020) 21(3):300-7.e2. doi: 10.1016/.j.jamda.2019.12.012

41. Chiles Shaffer N, Fabbri E, Ferrucci L, Shardell M, Simonsick EM, Studenski S. Muscle Quality, Strength, and Lower Extremity Physical Performance in the Baltimore Longitudinal Study of Aging. J Frailty Aging (2017) 6(4):183-7. doi: $10.14283 /$ jfa.2017.24

42. Cesari M, Leeuwenburgh C, Lauretani F, Onder G, Bandinelli S, Maraldi C, et al. Frailty Syndrome and Skeletal Muscle: Results From the Invecchiare in Chianti Study. Am J Clin Nutr (2006) 83(5):1142-8. doi: 10.1093/ajcn/ 83.5.1142

43. Colleluori G, Villareal DT. Aging, Obesity, Sarcopenia and the Effect of Diet and Exercise Intervention. Exp Gerontol (2021) 155:111561. doi: 10.1016/ j.exger.2021.111561

44. Tizazu AM, Nyunt MSZ, Cexus O, Suku K, Mok E, Xian CH, et al. Metformin Monotherapy Downregulates Diabetes-Associated Inflammatory Status and Impacts on Mortality. Front Physiol (2019) 10(572). doi: 10.3389/ fphys.2019.00572

45. Zamboni M, Nori N, Brunelli A, Zoico E. How Does Adipose Tissue Contribute to Inflammageing? Exp Gerontol (2021) 143:111162. doi: 10.1016/j.exger.2020.111162

46. Bellary S, Kyrou I, Brown JE, Bailey CJ. Type 2 Diabetes Mellitus in Older Adults: Clinical Considerations and Management. Nat Rev Endocrinol (2021) 17(9):534-48. doi: 10.1038/s41574-021-00512-2
47. Sergi D, Naumovski N, Heilbronn LK, Abeywardena M, O'Callaghan N, Lionetti L, et al. Mitochondrial (Dys)function and Insulin Resistance: From Pathophysiological Molecular Mechanisms to the Impact of Diet. Front Physiol (2019) 10:532. doi: 10.3389/fphys.2019.00532

48. Andreux PA, van Diemen MPJ, Heezen MR, Auwerx J, Rinsch C, Groeneveld GJ, et al. Mitochondrial Function is Impaired in the Skeletal Muscle of PreFrail Elderly. Sci Rep (2018) 8(1):8548. doi: 10.1038/s41598-018-26944-x

49. Coen PM, Musci RV, Hinkley JM, Miller BF. Mitochondria as a Target for Mitigating Sarcopenia. Front Physiol (2019) 9:1883. doi: 10.3389/ fphys.2018.01883

50. Pramyothin P, Limpattanachart V, Dawilai S, Sarasak R, Sukaruttanawong C, Chaiyasoot K, et al. Fat-Free Mass, Metabolically Healthy Obesity, and Type 2 Diabetes in Severely Obese Asian Adults. Endocr Pract (2017) 23(8):915-22. doi: 10.4158/EP171792.OR

51. Kozakowski J, Gietka-Czernel M, Leszczyńska D, Majos A. Obesity in Menopause - Our Negligence or an Unfortunate Inevitability? Prz Menopauzalny (2017) 16(2):61-5. doi: 10.5114/pm.2017.68594

52. Mat S, Tan MP, Mohktar MS, Kamaruzzaman SB, Ibrahim F. Phase Angle and Diabetes in Community-Dwelling Older Adults: Cross-Sectional Analysis From the Malaysian Elders Longitudinal Research (MELoR) Study. Eur J Clin Nutr (2021). doi: 10.1038/s41430-021-01020-2

53. Rondanelli M, Talluri J, Peroni G, Donelli C, Guerriero F, Ferrini K, et al. Beyond Body Mass Index. Is the Body Cell Mass Index (BCMI) a Useful Prognostic Factor to Describe Nutritional, Inflammation and Muscle Mass Status in Hospitalized Elderly?: Body Cell Mass Index Links in Elderly. Clin Nutr (2018) 37(3):934-9. doi: 10.1016/j.clnu.2017.03.021

54. Volpato S, Bianchi L, Lauretani F, Lauretani F, Bandinelli S, Guralnik JM, et al. Role of Muscle Mass and Muscle Quality in the Association Between Diabetes and Gait Speed. Diabetes Care (2012) 35(8):1672-9. doi: 10.2337/dc11-2202

55. Baskaran D, Aparicio-Ugarriza R, Ferri-Guerra J, Milyani R, Florez H, Ruiz JG. Is There an Association Between Metformin Exposure and Frailty? Gerontol Geriatr Med (2020) 6:2333721420924956. doi: 10.1177/ 2333721420924956

56. Wu C-N, Tien K-J. The Impact of Antidiabetic Agents on Sarcopenia in Type 2 Diabetes: A Literature Review. J Diabetes Res (2020) 2020:9368583. doi: $10.1155 / 2020 / 9368583$

57. Wang M, Tan Y, Shi Y, Wang X, Liao Z, Wei P. Diabetes and Sarcopenic Obesity: Pathogenesis, Diagnosis, and Treatments. Front Endocrinol (Lausanne) (2020) 11:568. doi: 10.3389/fendo.2020.00568

58. Sezgin D, O'Donovan M, Woo J, Bandeen-Roche K, Liotta G, Fairhall N, et al. Early Identification of Frailty: Developing an International Delphi Consensus on Pre-Frailty. Arch Gerontol Geriatr (2021) 99:104586. doi: 10.1016/ j.archger.2021.104586

59. Izquierdo M, Merchant RA, Morley JE, Anker SD, Aprahamian I, Arai H, et al. International Exercise Recommendations in Older Adults (ICFSR): Expert Consensus Guidelines. J Nutr Health Aging (2021) 25(7):824-53. doi: 10.1007/ s12603-021-1665-8

Conflict of Interest: The authors declare that the research was conducted in the absence of any commercial or financial relationships that could be construed as a potential conflict of interest.

Publisher's Note: All claims expressed in this article are solely those of the authors and do not necessarily represent those of their affiliated organizations, or those of the publisher, the editors and the reviewers. Any product that may be evaluated in this article, or claim that may be made by its manufacturer, is not guaranteed or endorsed by the publisher.

Copyright (c) 2022 Merchant, Soong and Morley. This is an open-access article distributed under the terms of the Creative Commons Attribution License (CC BY). The use, distribution or reproduction in other forums is permitted, provided the original author(s) and the copyright owner(s) are credited and that the original publication in this journal is cited, in accordance with accepted academic practice. No use, distribution or reproduction is permitted which does not comply with these terms. 